\title{
Evaluación basada en los Resultados de Aprendizaje: Una Experiencia en la Universidad
}

\section{Learning Outcomes based Assessment: An Experience at University}

\author{
Eugenio Astigarraga Echeverría ${ }^{1}$ * \\ Arantza Mongelos García ${ }^{1}$ \\ Xavier Carrera Farran ${ }^{2}$ \\ ${ }^{1}$ Mondragon Unibertsitatea, España \\ ${ }^{2}$ Universitat de Lleida, España
}

\begin{abstract}
Partiendo de los cambios implementados en la Facultad de Humanidades y Ciencias de la Educación (HUHEZI) de Mondragon Unibertsitatea así como de los propiciados por el desarrollo del Espacio Europeo de Educación Superior (EEES), en este artículo se presenta una experiencia de innovación educativa -en cuanto a redefinición curricular, metodológica y evaluativa- desarrollada en los Grados de Educación Infantil y Educación Primaria de esta Facultad en los cursos 2017-18 (1 $\left.{ }^{\circ}\right)$ y 2018-19 ( $1^{\circ}$ y $\left.2^{\circ}\right)$ con una participación de 12 tutores, 40 profesores y 365 alumnos. Se fundamenta cómo, tras los cambios curricular y metodológicos habidos en la universidad, es necesario replantear el sistema de evaluación, acorde con dichas transformaciones y con las tendencias actuales de evaluación en Educación Superior. Se detalla la estrategia de evaluación diseñada a partir del perfil profesional y de las competencias a desarrollar en ambos grados y que se articula alrededor de los Resultados de Aprendizaje. Se presenta, con detalle, cómo se implementa el nuevo modelo evaluativo en cuanto a los momentos en que se lleva a cabo, los procedimientos y acciones requeridas, los agentes implicados, los instrumentos utilizados, las interacciones y feedbacks proporcionados, y los criterios evaluativos y de obtención de calificaciones establecidos. Finalmente, y sin intención de obtener generalizaciones -al tratarse de una experiencia que está en proceso de desarrollo-, se reflexiona sobre los aspectos y temáticas clave que pueden facilitar -o bien dificultar- el asentamiento y consolidación de las transformaciones que este tipo de innovación impulsan.
\end{abstract}

Palabras Clave: Evaluación; Innovación educativa; Resultados de aprendizaje; Universidad.

Based on the changes implemented at the Faculty of Humanities and Education Sciences (HUHEZI) in Mondragon Unibertsitatea, as well as those promoted by the development of the European Higher Education Area (EHEA), an experience of educational innovation - in terms of curricular, methodological and evaluative redefinition - is presented. The present experience was developed in the Degrees of Infant Education and Primary Education in this Faculty during the academic years 2017-18 (Year 1) and 2018-19 (Years 1 and 2) with a participation of 12 tutors, 40 teachers and 365 students. It shows how, after the curricular and methodological changes implemented in the university, it is necessary to rethink the evaluation system, in accordance with those transformations and with the current trends of evaluation in higher education. The evaluation strategy described, based on the professional profile and competencies to be developed in both degrees, is designed and articulated around the corresponding learning outcomes. It is presented, in detail, how the new evaluation model is implemented in terms of when it is carried out, the procedures and actions required, the agents involved, the instruments used, the interactions and feedback given, together with the evaluation and qualification criteria established. Finally, and without aiming to seek generalizations - since this is a particular experience that is still in process of development - we seek to reflect on the key aspects and themes that can facilitate -or hinder- the settlement and consolidation of the transformations that this type of innovation drives.

Keywords: Assessment; Educational innovation; Learning outcomes; University.

*Contacto: eastigarraga@mondragon.edu

issn: $1989-0397$

www.rinace.net/riee/

https://revistas.uam.es/riee
Recibido: $\quad 3$ de septiembre de 2019

$1^{\text {a }}$ Evaluación: 8 de octubre de 2019

$2^{\text {a }}$ Evaluación: 15 de noviembre de 2019

Aceptado: $\quad 21$ de noviembre de 2019 


\section{Introducción}

Teniendo como antecedentes -a nivel local- el Proyecto Mendeberri y -a nivel internacional- la construcción del Espacio Europeo de Educación Superior, iniciamos en la Facultad de Humanidades y Ciencias de la Educación de Mondragon Unibertsitatea ${ }^{1}$ un proceso de innovación curricular que, de forma resumida, puede consultarse en Ozaeta, Mongelos, Astigarraga y Garro (2018). Este proceso de rediseño de un nuevo currículum, se implementó el curso 2017-18 en el primer curso tanto del Grado de Educación Primaria como del Grado de Educación Infantil, y en el curso 2018-19 se ha extendido al segundo curso de ambos Grados de Educación.

El modelo competencial sobre el que se han desarrollado los diferentes trabajos ha buscado ser coherente con el Marco Europeo de Cualificaciones (EQF-MEC) y su adaptación al ámbito de la Educación Superior en el estado español mediante el Marco Español de Cualificaciones para la Educación Superior (MECES), que se estableció a través del Real Decreto 1027/2011, y fue posteriormente modificado mediante el Real Decreto 96/2014. A través del establecimiento de los diferentes Marcos de Cualificaciones (CEDEFOP, 2018) el modelo de formación basada en competencias toma como referente para su diseño, desarrollo y evaluación los Resultados de Aprendizaje -en adelante RA- (ANECA, 2013; CEDEFOP, 2017; CEDEFOP, 2019; Comisión Europea, 2009) modelo que en el estado español es apreciable de forma bien asentada en la Formación Profesional (Astigarraga y Carrera, 2018).

El cambio curricular realizado en HUHEZI parte de revisar y ajustar el perfil de salida del alumnado tomando como referentes las principales Funciones Profesionales y Competencias asociadas a las mismas. En base a este reajuste se establecen los $\mathrm{RA}^{2}$, que son la guía para la definición de las actividades de aula (Ozaeta et al., 2018). Toda esta transformación, dirigida desde un grupo dinamizador, se desarrolla con la participación abierta del conjunto del profesorado implicado en los respectivos Grados de Educación. El producto resultante -RA para el Grado en su conjunto y para cada uno de los cursos del mismo (véase figura 1) - requiere tiempo y trabajo colaborativo, que además de la obtención del propio producto, tiene como finalidad la mejora y el crecimiento profesional de todos los docentes de la Facultad, a la par que se orienta hacia la mejora de la actividad docente en la línea que señala Hattie (2017) "la planificación conjunta de las lecciones es la tarea con una de las más altas probabilidades de establecer una marcada diferencia positiva en el aprendizaje del alumno" (p. 95). El pasar del yo al nosotros, del trabajo individual/aislado del docente al trabajo en equipo de los docentes, es una tendencia que es cada vez más requerida (Guerriero, 2017; Paniagua e Istance, 2018), y que en nuestro contexto está resultando efectiva y clave para el cambio y la innovación en las instituciones

\footnotetext{
${ }^{1}$ En adelante HUHEZI, por sus siglas en euskera (lengua vehicular y de comunicación de esta Facultad).

2 Desde el CEDEFOP (2017: 29) se diferencia -si bien se subraya su interrelación- entre Resultados de Aprendizaje Pretendidos (Intended Learning Outcomes) y Resultados de Aprendizaje Logrados (Achieved Learning Outcomes); a nuestros efectos, en la planificación nos referimos, obviamente, a los primeros.
} 
educativas, tal como se evidencia a partir de la investigación realizada el presente año por Sarobe, López-Salas y Astigarraga (2019).

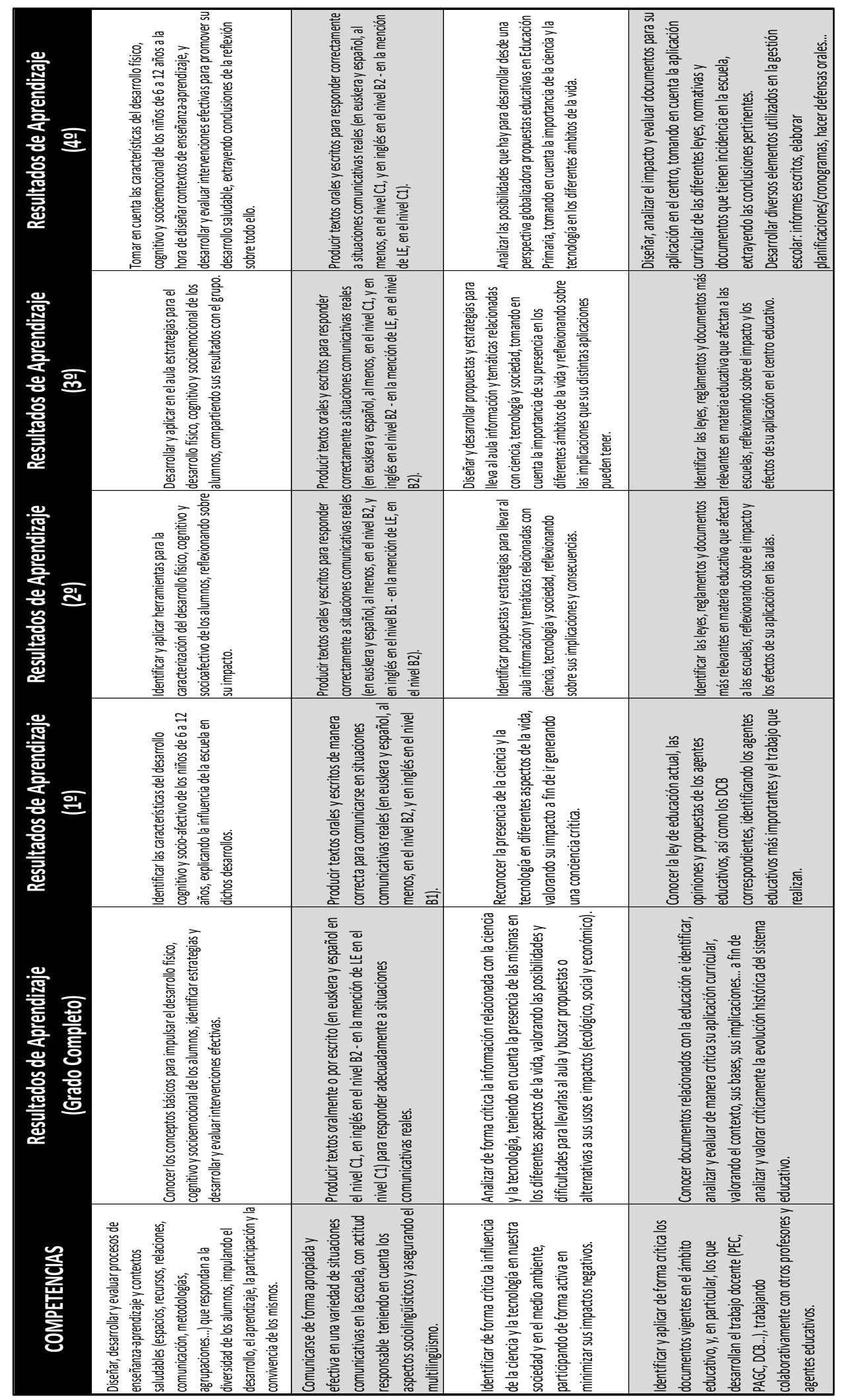

Figura 1. Ejemplos de Secuencia de: Competencia - Resultados de Aprendizaje para el Grado - Resultados de Aprendizaje por Cursos Fuente: Elaboración propia. 
De esta manera, constatamos que si bien el trabajo participativo y colaborativo en el ámbito curricular puede iniciarse desde alguno de sus componentes, debemos anticipar que va a afectar al conjunto del ecosistema que es la institución educativa. En este sentido es clarificadora la postura de Scott (2015a) cuando señala (mencionando a Trilling y Fadel, 2009, p. 115) que "investigaciones recientes indican que para normalizar el aprendizaje colaborativo será necesario introducir cambios en los planes de estudios, la docencia, las prácticas de evaluación, los entornos de aprendizaje y el desarrollo profesional de las y los docentes" (p. 5). Esta misma autora, en otro de sus trabajos para la UNESCO (Scott, 2015 b) nos remite a la necesidad de ampliar la perspectiva de la evaluación en los procesos de enseñanza-aprendizaje.

\section{Fundamentación: del cambio metodológico al cambio en la evaluación}

La perspectiva de la evaluación -en función del modelo/paradigma educativo en el que explícita o implícitamente se situaba el profesorado- ha ido cambiando a lo largo del tiempo, y, a menudo, se constituye en tema de amplios debates y difíciles acuerdos.

Desde finales de los años 80 del pasado siglo (Frey, Schmitt y Allen, 2012), se fue extendiendo la denominada evaluación auténtica, siendo su principal objetivo mejorar el aprendizaje de los alumnos implicándolos en los procesos de evaluación (Brown, 2015), para lo que debía cumplir con el principio de alineamiento constructivo de Biggs (2014) entre RA, actividades de evaluación y actividades de aprendizaje (figura 2).

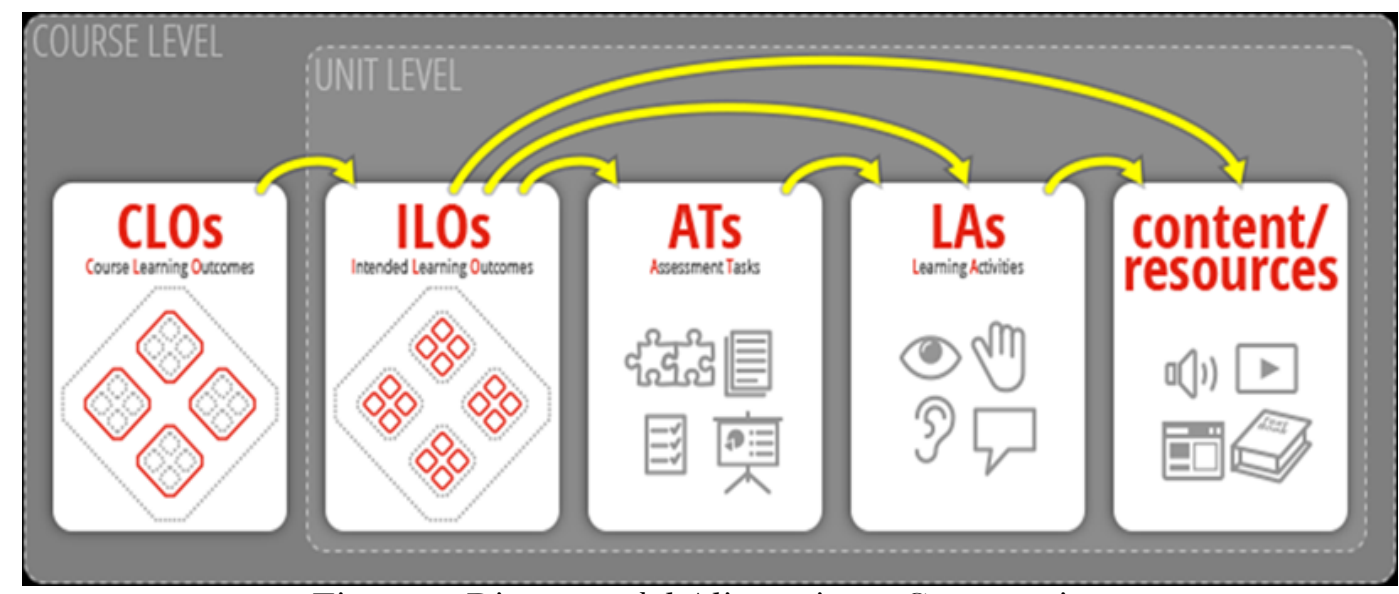

Figura 2. Diagrama del Alineamiento Constructivo

Fuente: Rundle y Gurney (2017).

En este contexto, Brown (2015) establece entre las principales características de la evaluación auténtica, las siguientes:

- Orientada a la acción en diversidad de contextos relevantes;

- Respaldada por evidencia relevante surgida de la práctica profesional;

- Matizada, articulada y transparente en la toma de decisiones;

- Verdaderamente representativa del esfuerzo y del rendimiento del estudiante; 
- Con criterios ponderados en función de su importancia en términos de los RA;

- Proclive a maximizar el esfuerzo y el tiempo dedicados a la tarea por parte de los docentes;

- Incluyente en sus planteamientos, de modo que permita que todos los estudiantes lleguen al máximo de lo que son capaces.

SantaCruz (2019), siguiendo a Biggs y Tang (2011), relaciona el alineamiento constructivo con el aprendizaje profundo (deep learning), al tiempo que indica que la puesta en práctica de un proceso de aprendizaje superficial (surface learning) es un indicador de la falta de alineamiento constructivo entre los tres elementos -RA, tareas de evaluación, actividades de aprendizaje- que intervienen en el mismo (figura 2), y concluye que, si bien "la transformación e innovación educativa no es una tarea fácil de lograr, el marco de alineamiento constructivo ayuda a los educadores docentes a reflexionar sobre sus propios diseños e implementaciones de enseñanza y aprendizaje” (SantaCruz, 2019, p. 7).

En este proceso reflexivo es importante una correcta identificación y definición de los RA, que indicarán a los estudiantes qué es lo que se considera importante de aprender, así como la secuencia y progresión que dichos aprendizajes van a seguir. Este trabajo de identificación y concreción de los RA requiere, tal como afirman Ozaeta et al. (2018), de la participación activa del conjunto del profesorado, situando dicha tarea en un marco de cambios educativos que acertadamente reflejan Siarova, Sternadel y Mašidlauskaitė (2017) al indicar que:

Los objetivos de aprendizaje ya no están exclusivamente asociados a habilidades relacionadas con una determinada disciplina académica. Se espera que la educación 'desarrolle las competencias de los individuos' para enfrentar problemas y demandas complejas, movilizando recursos psicosociales, conocimientos, habilidades y actitudes adquiridas previamente en situaciones de aprendizaje similares a los contextos que se les presentarán en su vida diaria, profesional o académica (p. 27).

Así pues, los RA, además de señalar los objetivos a desarrollar por los equipos docentes, ofrecen pistas de gran interés en relación con los contenidos a desarrollar -asociados a los distintos tipos de competencias-, con las formas de trabajo en el aula -pertinentes y válidas para el desarrollo y logro de todo ello-, así como con los espacios o entornos de aprendizaje en los que los procesos educativos deben desarrollarse -lo más parecidos y cercanos posibles a los contextos de aplicación de las competencias-.

Por otra parte, la ampliación de las competencias a desarrollar -incluyendo, junto a las competencias técnicas, las competencias del siglo XXI- genera dificultades en los procesos evaluativos; tal como señalan Siarova et al. (2017), "la evaluación de competencias clave y competencias transversales es un reto, ya que se refieren a constructos complejos que no son fácilmente medibles". Y añaden que "la evaluación de las competencias sociales y emocionales de los alumnos se realiza, generalmente, desde una perspectiva formativa [...] que todavía es menos transparente que la evaluación de los logros académicos” (p. 29).

Para Hill y Barber (2014) es necesario ajustar la evaluación como parte del proceso contínuo de repensar el aprendizaje y la enseñanza. Esta necesidad de repensar las formas de evaluación, para alinearlas con las actividades, los objetivos, las dinámicas de aula... es también un punto esencial del Proyecto Mendeberri 2025 en el que se subraya que "la evaluación es un tema central en cualquier cambio de paradigma educativo ya que está comprobado que difícilmente cambian los alumnos su forma de aprender si los 
aprendizajes conseguidos se evalúan en función de modelos evaluativos previos que no cambian” (García, Zubizarreta y Astigarraga, 2017, p. 51).

Si bien en estas últimas décadas se han ido extendiendo propuestas centradas en el enfoque de evaluación auténtica, el tema de la evaluación sigue siendo un amplio campo de controversia y de dificultades innegables (Pellegrino, 2017; Siarova et al., 2017) en el que se van consolidando algunas tendencias y prácticas de la evaluación que deberán ir contrastándose a lo largo del tiempo. En este sentido son clarificadoras las palabras de Pellegrino (2017) cuando afirma que, "si bien se ha progresado en la evaluación de las habilidades cognitivas, se necesita mucha más investigación para desarrollar evaluaciones de las habilidades interpersonales e intrapersonales que sean adecuadas para los usos formativos y sumativos de la evaluación en entornos educativos" (p. 245).

En síntesis, las tendencias actuales en la evaluación de las competencias en la Educación Superior requieren que el abordaje de la evaluación se haga desde múltiples miradas (sentido y funciones de la educación, metodologías, dinámicas de aula, rol de docentes y alumnos, objetivos...) lo que conlleva una visión poliédrica, sistémica y global de los procesos educativo-formativos que vamos a implementar con enfoques innovadores, así como de la sociedad en la que se desarrollan dichos procesos.

\subsection{Características de un nuevo marco para la evaluación en la universidad}

La definición y concreción de un marco para la evaluación, conlleva responder -de forma actualizada, integrada y pertinente- a preguntas ya clásicas, que podemos resumir en: ¿para qué evaluar?, ¿qué evaluar?, ¿cómo y cuándo evaluar?, ¿quién evalúa?, ¿qué uso se hace de la información obtenida en la evaluación? ...

Las respuestas a las preguntas anteriores pueden tener muy diversas respuestas en función del foco y finalidad(es) de la evaluación que se asuman y de los destinatarios de la formación. En el caso de la evaluación de los procesos de enseñanza-aprendizaje que se desarrollan en los Grados de Educación Infantil y Primaria, una de las funciones principales de la evaluación es la acreditación del logro de los mínimos exigidos para el desempeño docente.

Ahora bien, las tendencias señaladas anteriormente, la demanda de que los nuevos perfiles profesionales incorporen las competencias del siglo XXI -que no son exclusivas de esta profesión, ni tan siquiera del ámbito académico o laboral-, las organizaciones internacionales (OCDE, UNESCO, WEF), el propio proyecto Mendeberri 2025 ... nos obligan a plantearnos una evaluación que va más allá de la certificación. Es más, junto a la extendida idea de que el alumno ha de ser responsable de su aprendizaje (a lo largo de la vida), se va afianzando -como complemento imprescindible de/para la misma- que el alumno ha de asumir la responsabilidad de su evaluación.

En este sentido, constatamos que -actualmente (Lee, 2013; SantaCruz, 2019; Siarova et al., 2017)- los tres principales enfoques de la evaluación -no excluyentes entre sí- podemos mencionarlos (figura 3) como:

- Evaluación del aprendizaje (Assessment of learning)

- Evaluación para el aprendizaje (Assessment for learning)

- Evaluación como aprendizaje (Assessment as learning) 

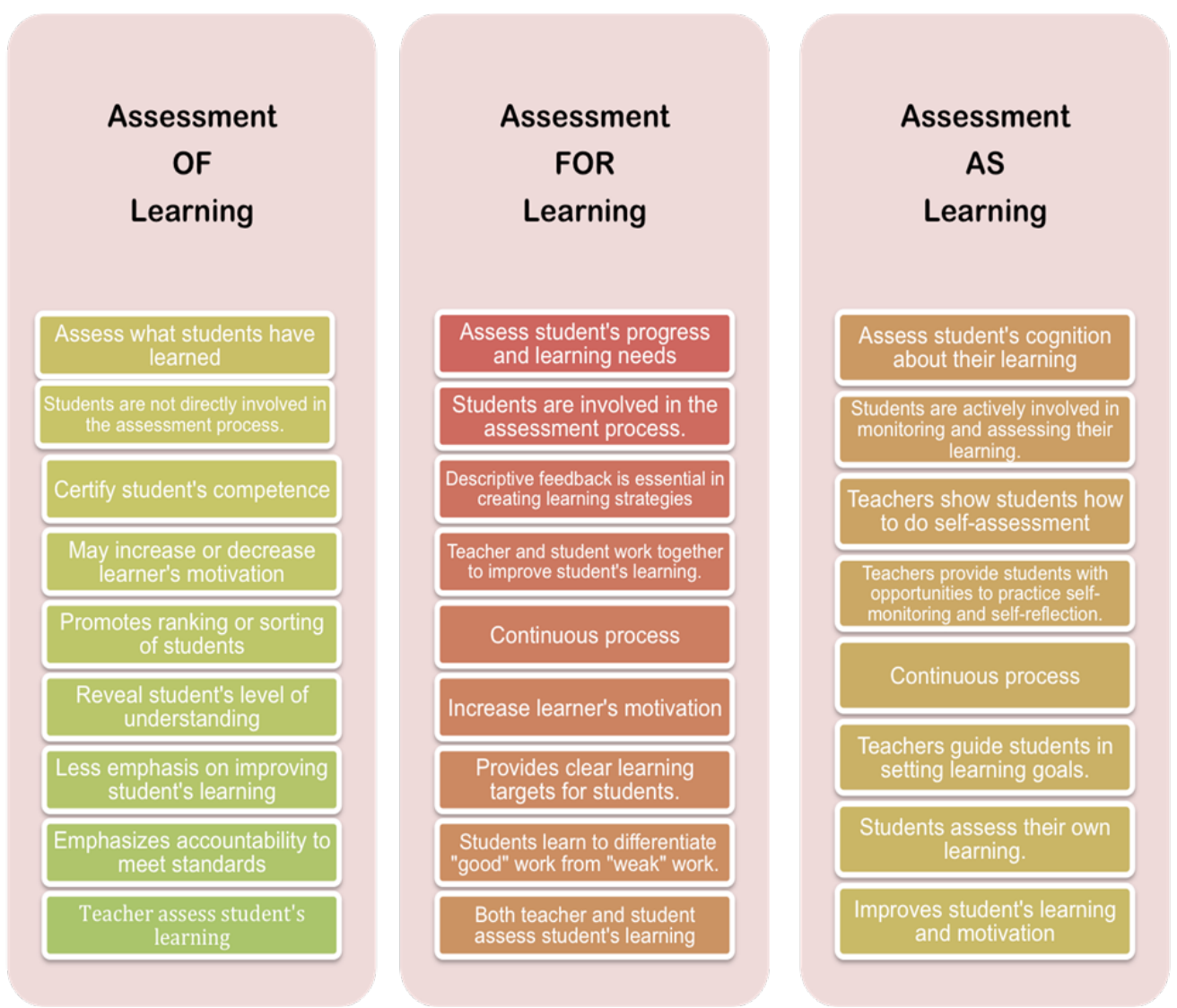

Figura 3. Evaluación del / para el / como aprendizaje

Fuente: Lee (2013).

El primero de estos enfoques se relaciona fácilmente con la evaluación sumativa y con la perspectiva certificadora de los procesos de enseñanza-aprendizaje. El segundo enfoque se puede asimilar -desde una perspectiva histórica- a los procesos de evaluación diagnóstica y, más específicamente, a la evaluación formativa. La combinación en la práctica de ambas formas de actuar ya conlleva cambios notables sobre los procesos de evaluación tradicionales que se han ido realizando en el ámbito universitario o en la Formación Profesional. En este último ámbito, ya se viene impulsando desde hace unos años este cambio hacia lo que se viene denominando evaluación como evolución (Tknika, 2019):

El enfoque Ethazi del proceso de evaluación de los alumnos es un enfoque basado en la evolución del grado de adquisición de sus competencias tanto técnicas como transversales, a medida que las ejercita en los sucesivos retos en los que trabajan con sus compañeros [...] La evaluación es un proceso que requiere de la participación de todos los actores: profesores y alumnos, principalmente, pero también personas del exterior (expertos, tutores de empresa...).

Cuando se estima oportuno, se desarrolla un proceso de evaluación a 360 grados donde tanto profesores como alumnos y profesionales pueden opinar sobre el grado de adquisición de las competencias de cada alumno. Fruto de estas evaluaciones multi-enfoque se va ofreciendo - a lo largo del proceso- el feedback necesario, que posibilita la mejora de los procesos que se están desarrollando en el contexto del reto. También con ello, se obtienen unos resultados que son ofrecidos a cada alumno y/o a cada equipo en un formato que invite a la reflexión, y son acompañados por un proceso de feedback (de final de reto) por parte de los profesores, cuyo objetivo es obtener unos compromisos y acciones de mejora por parte del alumno/equipo de cara a la ejecución de los siguientes retos (p. 4). 
El tercero de los enfoques -también en un contexto de evaluación formativa, pero, orientada así mismo a constatar los logros adquiridos-, permite facilitar al alumno el proceso de aprendizaje sobre su propia evaluación, de manera que el aprender a aprender se desarrolle también en relación con la evaluación de su propio proceso formativo y del logro de sus aprendizajes. Este proceso de metacognición debe ser facilitado y guiado por los docentes, los tutores... en definitiva, en el ámbito académico, por todos aquellos que participan en el proceso educativo-formativo del alumnado.

Con todo, esta aproximación por partes a la evaluación no debe perder su carácter de visión conjunta orientada al aprendizaje. Tal como señala Hayward (2015):

Las preposiciones que vinculan la evaluación con el aprendizaje, como, para y de, pueden ser útiles si enfocan la atención en diferentes propósitos para la evaluación. Sin embargo, existe el peligro de que estas preposiciones se conviertan en un mantra irreflexivo que distraiga la atención del constructo clave: la evaluación es el aprendizaje. Centrarse en la evaluación para el aprendizaje en las aulas es una condición necesaria pero no suficiente [...] debemos reconocer la evaluación como aprendizaje: las preposiciones, por lo tanto, pueden reflejar diferentes propósitos de evaluación, pero la razón de ser de la evaluación es el aprendizaje. (p. 38)

La puesta en práctica de lo mencionado anteriormente requiere disponer de momentos y estrategias que permitan desarrollar todo ello, pero, previamente, demanda identificar el qué se va a evaluar. Tal como se ha indicado en la introducción, nuestro referente de evaluación son los RA (Astigarraga y Carrera, 2018) derivados de las competencias identificadas (Ozaeta et al., 2018) previamente. El trabajo sobre los RA se ve facilitado por el uso de las taxonomías existentes en educación. En nuestro contexto son diversas las que podemos encontrar (Dreyfus, Anderson y Krathwohl, Marzano y Kendall...) siendo la más conocida y utilizada la taxonomía de Bloom. Desde nuestra perspectiva, y en consonancia con el enfoque de alineamiento constructivo y del contexto en que se desarrolla esta experiencia de innovación, se opta por utilizar la taxonomía SOLO de Biggs (2011, 2014).

\section{Contexto de la experiencia}

La Facultad de Humanidades y Ciencias de la Educación (HUHEZI) de Mondragon Unibertsitatea inició en el curso 2014-2015 (figura 4) un proceso de cambio que toma como eje el desarrollo de un currículum basado en competencias y operativizado por medio de "Propuestas de Trabajo" inspiradas en retos con una perspectiva inter/transdisciplinar (Ozaeta et al., 2018).

Este proceso de innovación educativa se inicia en el Grado de Comunicación Audiovisual, y se le da continuación en los grados de Educación Infantil y Educación Primaria. La experiencia de la Facultad en el desarrollo del Proyecto Mendeberri, y, posteriormente, en la adecuación de las titulaciones al Espacio Europeo de Educación Superior, sirve como referencia previa para avanzar en la definición y la aplicación de un nuevo modelo educativo más ajustado a las necesidades emergentes que presenta este siglo XXI.

El curso 2017-18 se inicia la aplicación de este nuevo modelo formativo y evaluativo participando en el mismo 2 grupos del Grado de Educación Infantil y 4 grupos del Grado de Educación Primaria; cada uno de los anteriores grupos tiene su tutor (6 tutores de curso) y participan -de forma parcial, en función de las distintas Propuestas de Trabajoun total de 30 docentes y cerca de 180 alumnos. 
Durante el curso 2018-19, han tomado parte en este proceso 4 grupos del Grado de Educación Infantil $\left(2\right.$ de $1^{\circ}$ y 2 de $2^{\circ}$ ) y 8 grupos del Grado de Educación Primaria ( 4 de $1^{\mathrm{o}}$ y 4 de $2^{\mathrm{o}}$ ). Cada uno de los anteriores grupos tiene su tutor (12 tutores de curso) y han participado -de forma parcial, en función de las distintas Propuestas de Trabajo- un total de 30 docentes en $1^{\mathrm{er}}$ curso y cerca de 40 en el $2^{\circ}$ curso. En cuanto al número de alumnos, han sido 190 alumnos en $1^{\text {er }}$ curso y 175 en $2^{\circ}$ curso.

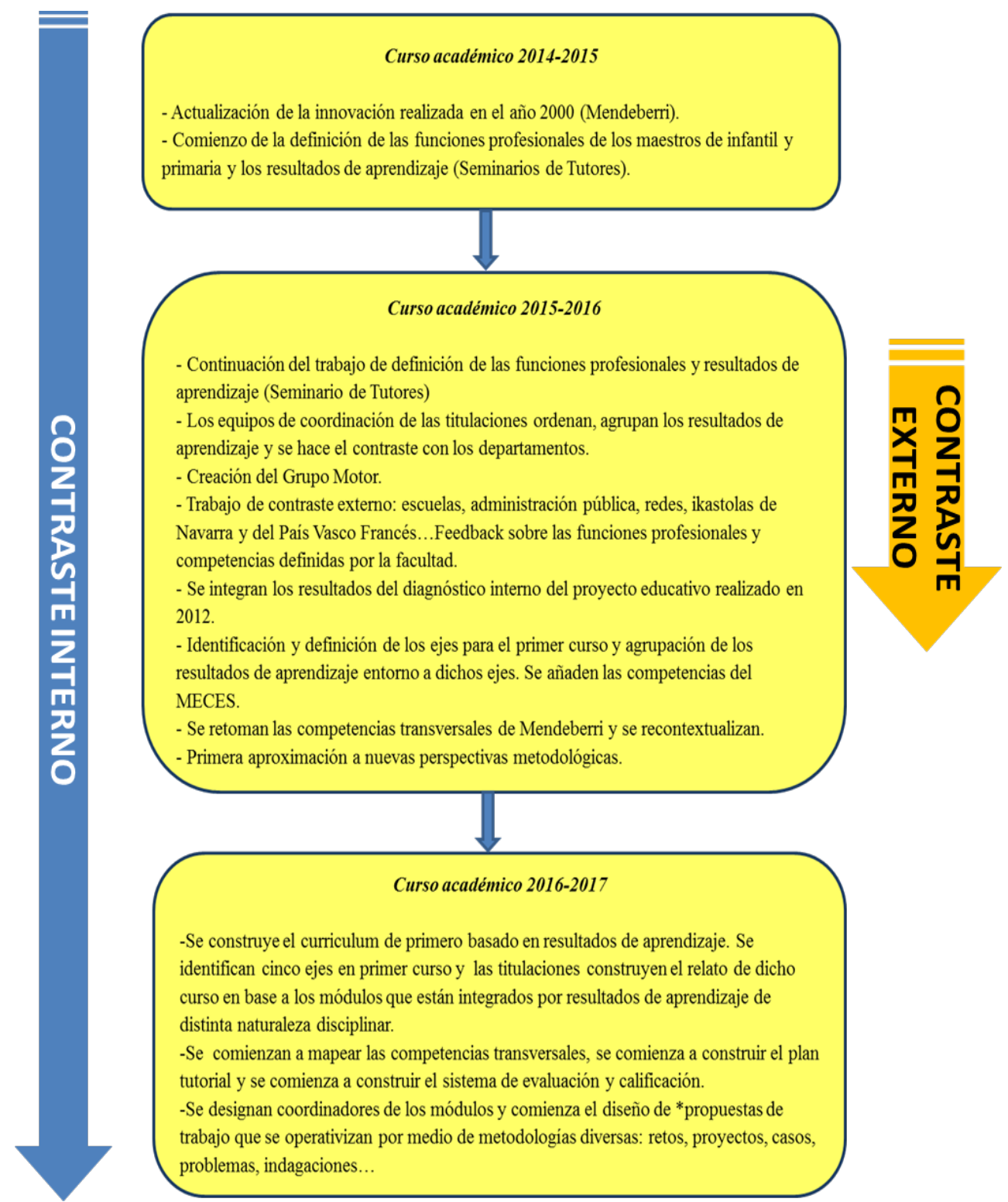

Figura 4. Proceso de rediseño curricular de los Grados de Educación Infantil y Educación Primaria

Fuente: Elaboración propia. 


\section{Diseño de la estrategia de evaluación en base a los Resultados de Aprendizaje}

En la perspectiva educativa que configura el marco para la evaluación definido en los puntos anteriores, la estrategia de evaluación se va definiendo a la par que se estructura la propuesta curricular para el aula. Tras haber llegado a la concreción de los RA (Ozaeta et al., 2018), se comienza a desplegar el proceso que se muestra de forma esquemática en la figura 5 .

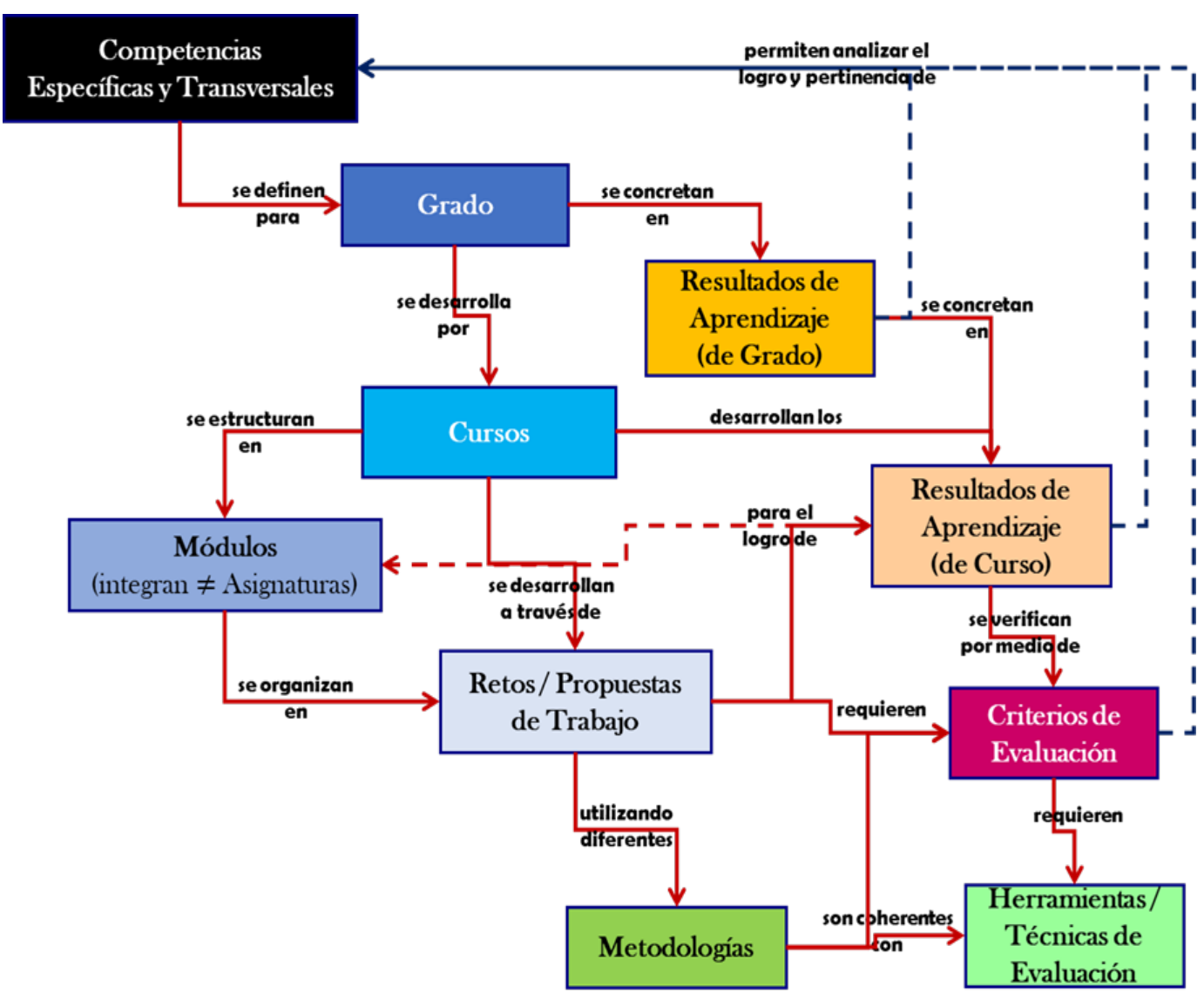

Figura 5. Proceso para el diseño curricular en base a los Resultados de Aprendizaje Fuente: Elaboración propia.

De esta manera, se procede a un trabajo en paralelo de: a) revisión y ajuste de los RA del primer curso y b) definición y concreción de los Módulos sobre los que soportar tanto los procesos curriculares (temáticas y contenidos a trabajar, alcance y delimitación de los mismos...) como los procesos administrativos (unidad mínima de matrícula, criterios para superación/repetición de curso...).

La primera de estas tareas lleva al conjunto del profesorado a especificar los RA para cada uno de los cursos de acuerdo a los niveles de la taxonomía SOLO, asumiendo que al finalizar los procesos de enseñanza-aprendizaje no tendríamos ningún alumno o alumna en el nivel preestructural. Un ejemplo de ello puede verse en la figura 6. A su vez, conlleva establecer cuál será el mínimo a lograr en cada curso. Normalmente, para cada RA el 
mínimo establecido en los distintos casos ha sido el nivel 2 (multiestructural), o el nivel 3 (relacional).

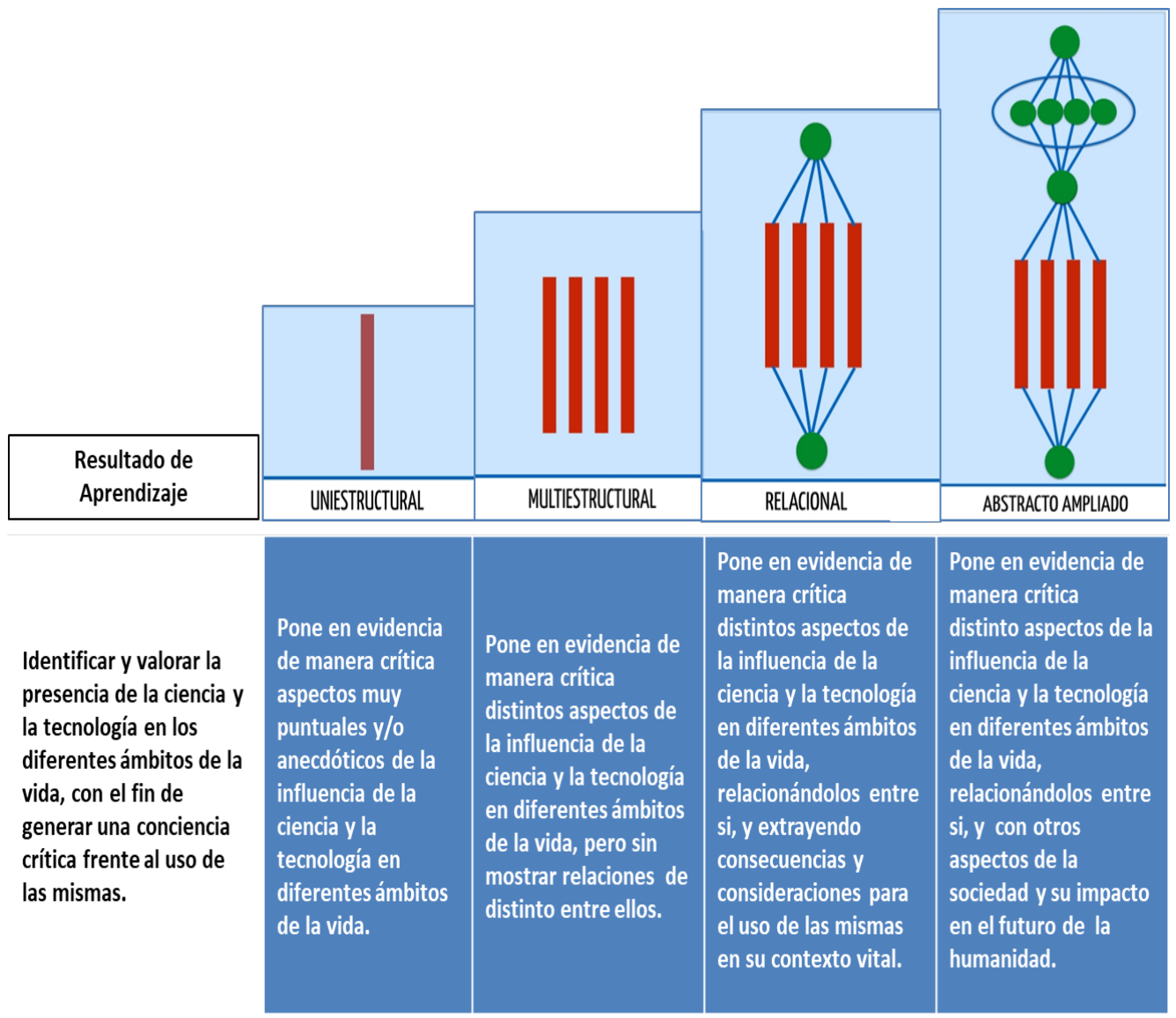

Figura 6. Ejemplo de nivelación de un Resultado de Aprendizaje

Fuente: Elaboración propia.

Todo este proceso de definición del marco de evaluación, de comprensión de la taxonomía a utilizar, de especificación y nivelación de los RA es complejo, pero asumimos que el mismo es imprescindible y permite una comprensión compartida de la evaluación por parte del conjunto del cuerpo docente. Tal como señalan Siarova et al. (2017) entre las condiciones "para garantizar prácticas de evaluación coherentes para el 'aprendizaje del siglo XXI', se encuentran:

- La importancia de una definición clara de las competencias de los estudiantes en términos de RA y su reflejo en el currículum;

- La formación docente, incluida la ITE [Initial Teacher Education], la etapa de inducción y la CPD [Continued Professional Development], que proporciona a los profesores una comprensión común de las competencias clave y la orientación de la evaluación a lo largo de sus carreras profesionales;

- Mecanismos de colaboración en forma de comunidades de enseñanza-aprendizaje (TLC Teaching and learning communities), con el objetivo de mejorar las prácticas de evaluación para alinearlas mejor con las competencias clave” (p. 45). 
En el desarrollo de los pasos anteriores, se van compartiendo y asumiendo diferentes principios que dirigirán los procesos de evaluación posteriores. Entre estos principios, los más significativos son los siguientes:

- El foco debe estar en los RA, siendo la labor de los docentes facilitar e impulsar su desarrollo por parte de los alumnos.

- El referente sobre el que se trabaja son los Móduloss, por lo que las Propuestas de Trabajo definidas dentro de los mismos no se calificarán.

- La evaluación debe ser pensada desde el Módulo en su globalidad, haciéndose el desarrollo y seguimiento de los RA desde el mismo.

- Para la calificación del Módulo se tomará en consideración el peso porcentual asignado a cada RA en el mismo.

- La calificación de los RA se realizará al finalizar el Módulo. Para esta calificación se tomarán en consideración las calificaciones que se han otorgado a cada RA en cada uno de los distintos Módulos en los que está presente en la evaluación.

- Los alumnos no recibirán calificaciones de los RA hasta la finalización del curso. De esta manera, al finalizar el curso tendrán dos calificaciones4: la correspondiente a cada uno de los RA y, en función de ello, la obtenida en cada uno de los Módulos.

- Dado que hay RA que se desarrollan en más de un Módulo, se compartirán las rúbricas de nivelación de los mismos.

- Desde cada Módulo se diseña y realiza el seguimiento de los alumnos en base a las correspondientes rúbricas de RA definidas previamente.

Continuando con el proceso de diseño, un paso posterior es el de concretar los RA a desarrollar desde cada uno de los Módulos. Esta tarea requiere de una visión global del curso y de acuerdos entre los diferentes equipos docentes que se constituyen para cada Módulo, y conlleva también una primera aproximación a la ponderación de los RA, tanto para su calificación como para la calificación final de los Módulos. Una primera aproximación de este trabajo puede verse en la figura 7 .

\footnotetext{
${ }^{3}$ Para el primer curso se definieron cinco Módulos. En el Grado de Educación Primaria fueron: I) La Escuela Inclusiva; II) Yo, futuro educador; III) Lengua, Pueblo y Escuela; IV) Ciencia y Tecnología en nuestra sociedad; V) Comunidad Educativa.

4 En realidad, son tres calificaciones ya que, por cuestiones administrativas, debemos seguir otorgando calificaciones a las asignaturas oficiales del plan de estudios. Esto se realiza a través de una conversión de la calificación de los Módulos en calificaciones de asignaturas mediante la correspondiente ponderación (en función de la presencia de cada asignatura en los distintos Módulos).
} 


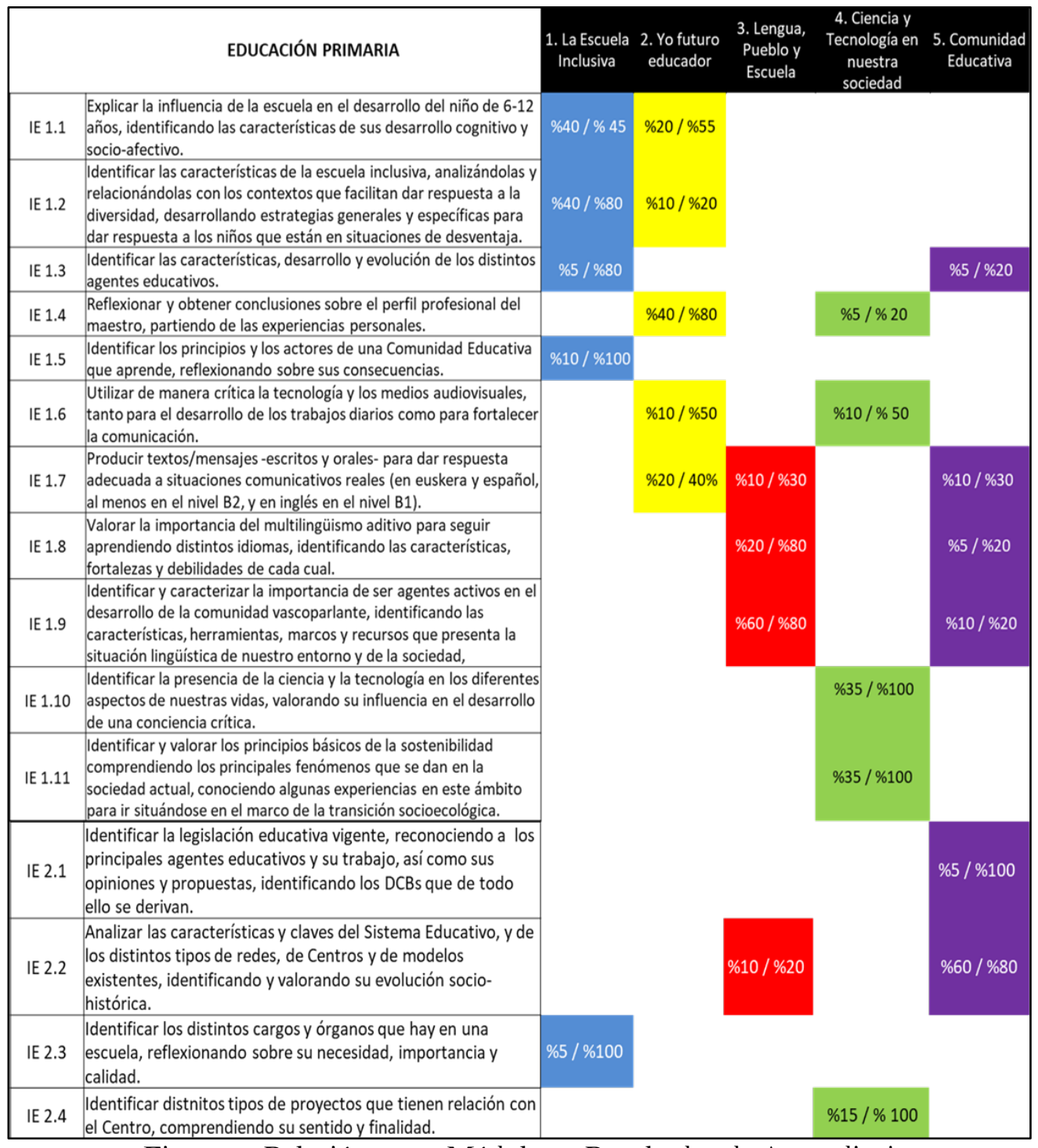

Figura 7. Relación entre Módulos y Resultados de Aprendizaje

Fuente: Elaboración propia.

A continuación, desde cada Módulo se identifican posibles Propuestas de Trabajo (PT), que utilizando diferentes Metodologías (Resolución de Problemas, Estudio de Casos, Proyectos, Conferencias, Visitas...) permiten desarrollar los RA correspondientes a cada Módulo (figura 8). Cada Módulo dispone de una Guía del Alumno que presenta tanto los RA a desarrollar en el mismo como las distintas Propuestas de Trabajo que lo posibilitarán; así mismo, junto al equipo de docentes que se hará cargo del mismo, se da a conocer de forma genérica el proceso de evaluación que se seguirá. De manera similar, se desarrolla una Guía del Alumno para cada una de las Propuestas de Trabajo, en la que se presentan con una mayor concreción las tareas a desarrollar así como los RA que se van a trabajar en dicha PT y los Criterios de Evaluación relacionados con cada uno de los RA a desarrollar; al mismo tiempo, además del cronograma correspondiente, se explicitan los agentes y las formas en que los mismos van a participar en la evaluación, siendo también tarea del equipo de profesores el desarrollo de las necesarias herramientas de evaluación para cada una de las Propuestas de Trabajo (en relación con los correspondientes Criterios 
de Evaluación $\left.{ }^{5}\right)$. Junto a estos procesos de evaluación, desde la Facultad, de manera genérica y periódica, se pasa también una encuesta de satisfacción (anónima) a los alumnos en relación con las PT desarrolladas.

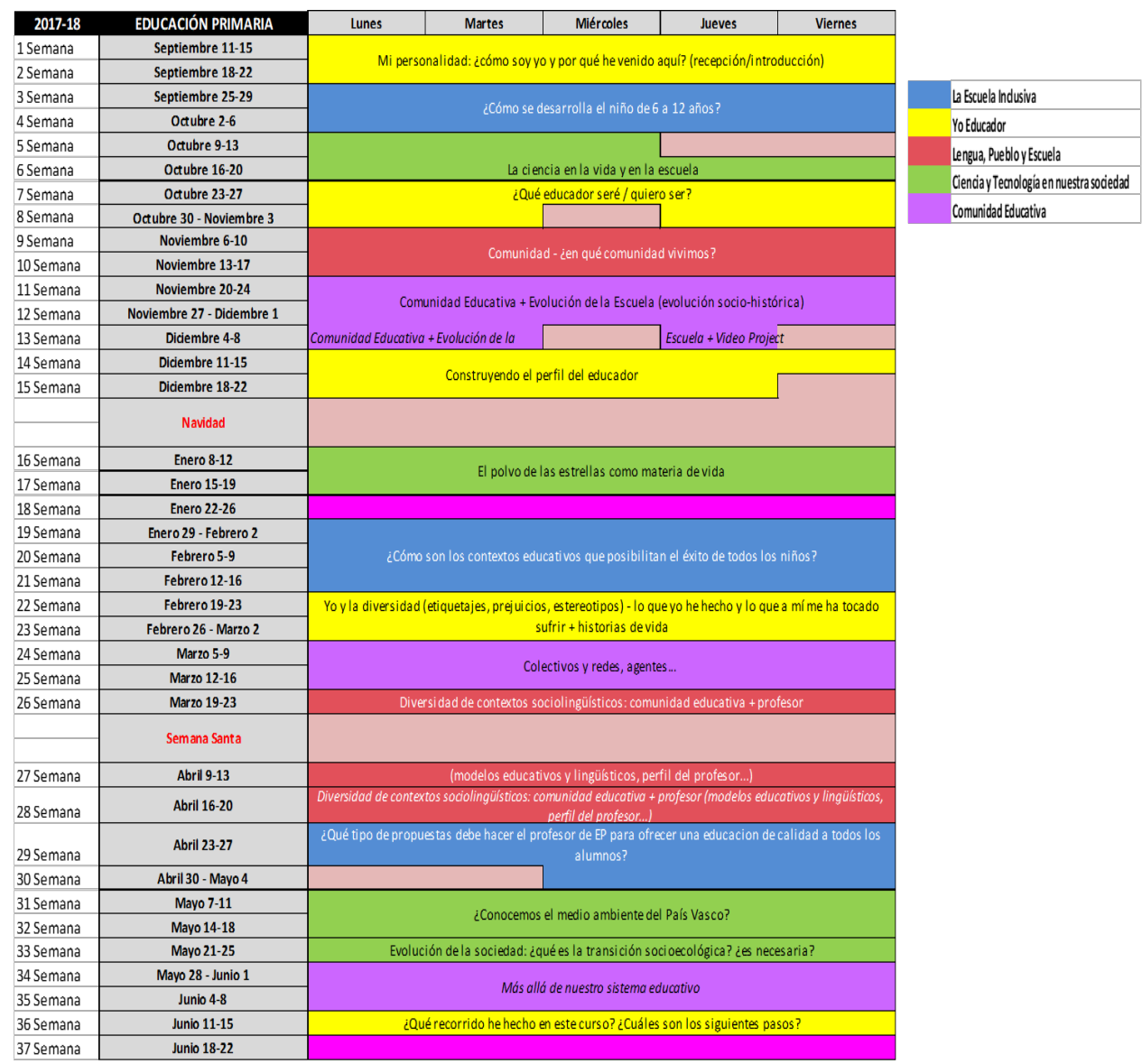

Figura 8. Módulos (dcha.) y Propuestas de Trabajo de cada uno de ellos (2017-18) Fuente: Elaboración propia.

\section{Desarrollo de la evaluación centrada en Resultados de Aprendizaje: el tutor como dinamizador}

Pasar del diseño y de la planificación mostrados en el apartado anterior requiere, ante todo, de la coordinación ágil y eficiente de todos los docentes que participan en el desarrollo de los RA a lo largo del curso con un mismo grupo de alumnos en los distintos

\footnotetext{
${ }^{5}$ Como puede entender el lector experimentado, este relato "tan lineal y sencillo" en la práctica es mucho más complejo (necesidad de compartir visiones de la educación y la evaluación; conocer y compartir metodologías y formas de trabajo en el aula; identificar y consensuar funciones de la evaluación, así como estrategias y técnicas para ello; acordar la ordenación y secuencia de las distintas Propuestas de Trabajo, etc.), y tampoco está finalizado para el primer día de comienzo del curso. Así pues, a menudo nos encontramos desarrollando una Propuestas de Trabajo y diseñando la(s) siguiente(s), por lo que es necesario subrayar que, además de trabajo y dedicación, hace falta una buena dirección, acompañamiento y ayuda a los docentes, así como una periódica revisión (normalmente a fin de curso) y mejora de los procesos desarrollados.
} 
módulos y en un mismo módulo. Así mismo, y a lo largo del proceso, es de vital importancia el seguimiento realizado al alumnado por parte del tutor de curso a lo largo de todo el año. Ambos elementos se explican a continuación, incidiendo en los momentos, instrumentos y objetivos de cada uno de ellos.

Al comienzo del curso, a cada estudiante se le asigna un tutor, que a su vez es el docente responsable del módulo "Yo Educador" en el primer año del grado. Este módulo es el de mayor presencia a lo largo de todo el curso, para poder realizar un mayor seguimiento y acompañamiento al alumno; y es el que da inicio y con el que se finaliza el curso académico de primero.

El tutor realiza, al menos, una reunión por trimestre con todos los docentes que participan en las propuestas de trabajo de los módulos. El objetivo de dichas reuniones es, principalmente, realizar un seguimiento cualitativo de los alumnos del grupo de forma que el tutor pueda ir orientando a cada uno de sus estudiantes según sus necesidades. Los docentes comparten sus experiencias, así como sus inquietudes y preocupaciones tanto desde la perspectiva de evolución de los estudiantes, como en relación con el desarrollo de los diferentes módulos (y los RA asociados a los mismos).

Además de estas reuniones, a lo largo del curso se realizan otras dos sesiones de "evaluación" propiamente dichas. Una en enero-febrero y la otra al finalizar el curso, en junio (en la figura 8, quedan recogidas estas semanas sin denominación específica). La primera de ellas sirve para que el equipo docente, por un lado, y los estudiantes, por otro, valoren cómo van progresando en los RA hasta el momento. Para ello, y de manera previa a la reunión de evaluación, cada docente realiza la valoración de los RA trabajados hasta la fecha en su módulo, y aplicando las rúbricas descritas anteriormente, le asigna el nivel correspondiente tomando en consideración los criterios de evaluación establecidos en cada una de las Propuestas de Trabajo desarrolladas hasta ese momento. El instrumento de evaluación -las distintas rúbricas- es compartido por todo el profesorado. Cada docente desarrolla y concreta su evaluación, y en la reunión de evaluación, coordinada por el tutor, se llega a acuerdos para cada alumno cuando se dan diferencias en las asignaciones del nivel de logro en un RA presente en dos o más módulos. En esta primera evaluación, únicamente se valora al estudiante teniendo en cuenta los niveles definidos en las rúbricas, no se obtiene ninguna calificación.

Al mismo tiempo, cada estudiante realiza su propia auto-evaluación de los RA. Para ello, cuenta con una herramienta específica en la que además de indicar el nivel en el que se encuentra -teniendo en cuenta la rúbrica para cada RA-, el estudiante tiene que evidenciar y justificar la valoración que realiza. En esta primera valoración, puede darse el caso de que algunos RA aún no se hayan podido empezar a desarrollar y queden sin valorar.

Realizadas ambas valoraciones, el tutor/a tiene una sesión de seguimiento con cada estudiante, en la que comparte la valoración realizada por los docentes y la realizada por el propio estudiante, contrastándolas con los niveles mínimos establecidos para cada uno de los RA. Ello da como resultado un informe valorativo y una gráfica (figura 9) que sintetiza las diferentes valoraciones obtenidas (valoración del docente, mínimo estipulado, autoevaluación del alumno). 


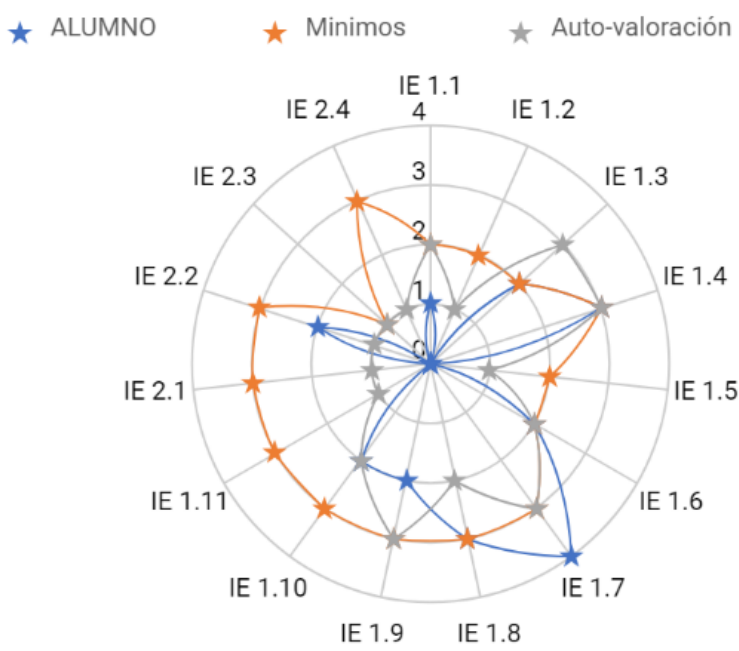

Figura 9. Síntesis gráfica de la primera valoración del progreso del alumno Fuente: Elaboración propia.

La sesión de tutoría tiene una duración de 30 minutos aproximadamente. El objetivo de la misma es compartir con cada estudiante la perspectiva del equipo docente con la que él/ella misma pueda tener de su progreso hasta el momento. En la misma sesión se acuerdan los objetivos de progreso para la segunda mitad de curso, analizando para ello las dificultades que se hayan podido detectar en cada caso, bien por parte del equipo docente bien por parte del propio alumno.

Al finalizar el curso, se realiza un proceso semejante de evaluación. En este caso, además de las valoraciones de cada uno de los RA asignados a cada módulo, los docentes han de calificar cada uno de dichos RA.

Para asignar las calificaciones, se han establecido unos baremos de forma que cada nivel tiene asignado un intervalo numérico, que permite diferenciar y precisar los logros de los alumnos aun cuando estén en el mismo nivel para un determinado RA, tal como se puede ver en la figura 10.

\begin{tabular}{|c|c|}
\hline Mínimo = 1 & \\
\hline Nivel & Calificación \\
\hline 1 & $5,0-5,9$ \\
\hline 2 & $6-7,4$ \\
\hline 3 & $7,5-8,9$ \\
\hline 4 & $9,0-10,0$ \\
\hline
\end{tabular}

\begin{tabular}{|c|c|}
\hline Mínimo = 2 \\
\hline Nivel & Calificación \\
\hline 2 & $5,0-6,9$ \\
\hline 3 & $7,0-8,5$ \\
\hline 4 & $8,6-10,0$ \\
\hline
\end{tabular}

Mínimo = 3

\begin{tabular}{|c|c|}
\hline Nivel & Calificación \\
\hline 3 & $5,0-7,9$ \\
\hline 4 & $8,0-10,0$ \\
\hline
\end{tabular}

Figura 10. Baremación por niveles en RA Fuente: Elaboración propia.

La sesión de evaluación de junio, al igual que la primera de enero-febrero, sirve para acordar los niveles de consecución de cada uno de los RA, y en caso necesario adecuar la calificación. Se entiende que aun cuando el nivel de consecución del RA ha de ser el mismo en el caso de RA compartidos por distintos módulos, las calificaciones no tienen por qué 
ser las mismas, siempre y cuando queden dentro de las franjas establecidas para cada nivel. Tras la evaluación, el tutor/a vuelve a tener una sesión de tutoría con cada alumno. En ella se contrastan las valoraciones realizadas por él mismo (mediante una herramienta similar a la utilizada en la sesión previa de enero, y en la que valora cada RA y justifica con evidencias dicha valoración) y las realizadas por el equipo docente con los mínimos establecidos. Los objetivos de esta tutoría son compartir con cada estudiante su situación con respecto a los RA a conseguir en el curso. En esta sesión el alumno, contrasta su progreso desde enero hasta junio (figura 11), posibilitando una aproximación a su calificación. Todos los alumnos acceden a las calificaciones una vez terminadas todas las tutorías a través de la secretaría virtual. Cada estudiante puede ver sus calificaciones para cada RA, así como para cada módulo, y finalmente, para cada asignatura.

En el transcurso de la sesión de tutoría, el estudiante y el tutor establecen las actividades a realizar para superar los RA no logrados, en caso de que los haya, así como objetivos de cara al siguiente curso en el que va a tener continuidad el desarrollo de los RA trabajados hasta el momento ${ }^{6}$. Estos compromisos quedan recogidos en su herramienta de evaluación y los retoma al comienzo del nuevo curso.

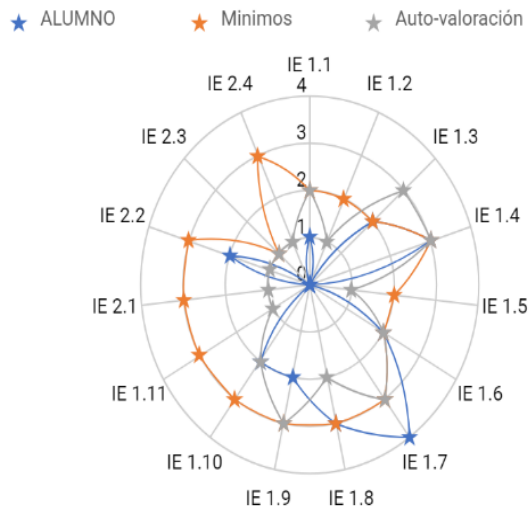

Enero

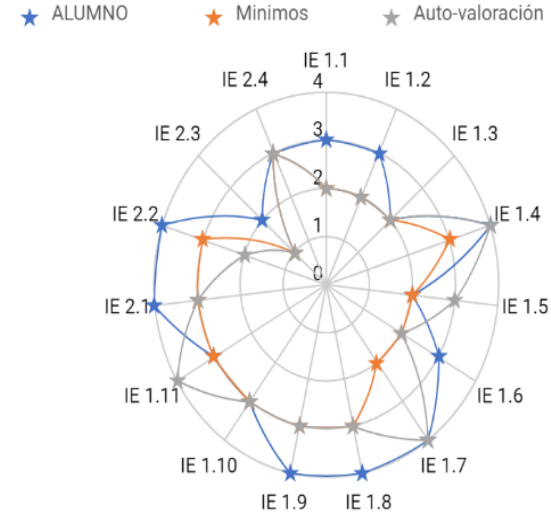

Junio

Figura 11. Síntesis gráfica de la progresión del alumno en su desarrollo de los RA Enero (izda.) / Junio (dcha.)

Fuente: Elaboración propia.

\section{Limitaciones y dificultades observadas}

Una reflexión crítica del desarrollo de la experiencia presentada nos lleva a clasificar las diferentes necesidades y dificultades que trae consigo el cambio curricular (objetivos, metodología, evaluación, rol docente...) en dos grandes ámbitos. El primero, relativo al diseño, conceptualización y visualización de los cambios que se quieren implementar. El segundo, relacionado con su puesta en práctica, seguimiento y consolidación.

\footnotetext{
${ }^{6}$ Como es lógico, puede haber algún RA nuevo, o bien puede darse que algún RA haya finalizado su proceso de desarrollo.
} 
En relación con el primer ámbito, los aspectos más reseñables son los siguientes. En primer lugar, observamos que -en nuestro contexto, y por mucho que se verbalice o aparezca en los documentos administrativos- no hay una comprensión compartida de qué es la Formación Basada en Competencias (FBC), y, unido a ello, la orientación a los RA que, en el contexto anglosajón, es más habitual. Por tanto, una dificultad y una tarea inicial es la de compartir en qué consiste un modelo de FBC, cómo se estructura el mismo, qué tipo de competencias lo incluyen... León (2014), citando a Flores (2003), recoge bien la esencia de este tipo de formación al señalar que:

Los modelos educativos por competencias están sustentados en tres ejes: la educación basada en competencias, la flexibilidad curricular y los procesos educativos centrados en el aprendizaje. Esto conduce a que "las prácticas educativas estén orientadas hacia la interdisciplinariedad, el trabajo grupal, el conocimiento aplicado a realidades concretas, el papel del docente como coordinador y facilitador del aprendizaje y la participación activa del estudiante en su proceso de formación (p. $55)$.

Unido a lo anterior, otra gran dificultad que se nos ha presentado es doble: cómo identificar y describir los RA, a la par que diferenciamos los mismos de las competencias. Esta cuestión es importante ya que el describir adecuadamente los RA esperados debe permitir al equipo docente pensar más allá de la propia asignatura, reflexionar sobre los aportes que la misma hace al logro de dichos RA, al tiempo que facilita el diseño de Propuestas de Trabajo para el aula de carácter interdisciplinar y referenciadas en el contexto profesional para el que se está formando al grupo de alumnos.

Ya en su momento, también desde la ANECA (2013) se señalaban algunas de las dificultades y limitaciones que conllevaba el trabajar con los RA, y en particular subrayaba que:

Definir el plan de estudios en términos de $R A$ requiere dedicación, esfuerzo, recursos y obstáculos a sortear. El cambio del enfoque hacia un modelo centrado en el estudiante exige la concienciación del personal académico de las universidades, la familiarización con su uso y la dedicación de su tiempo y esfuerzo para reflexionar sobre los resultados que deben lograr los estudiantes, así como su trabajo en equipo para lograr objetivos comunes e integrados en un nivel superior. Supone, por tanto, en muchas ocasiones una transformación significativa que a menudo tarda años en hacerse efectiva (p. 18).

Otra dificultad se presenta a la hora de concretar la evaluación desde las diferentes Propuestas de Trabajo. Aquí también la dificultad es doble. Por una parte, describir Criterios de Evaluación relacionados con las tareas a desarrollar y orientados a la evaluación de los RA, es la esencia en la práctica del alineamiento constructivo... pero no es siempre tarea fácil. Por otra parte, que un mismo RA se trabaje desde Propuestas de Trabajo desarrolladas por equipos de profesores distintos, conlleva la necesidad de una comprensión compartida de dicho RA, a la par que se acota la misma, dando la posibilidad de que cada Propuesta de Trabajo tenga sus propios Criterios de Evaluación orientados a RA compartidos. Esto requiere mucha coordinación y seguimiento, a la vez que resulta imprescindible dar apoyo a los distintos equipos de docentes que están diseñando y desarrollando las diversas Propuestas de Trabajo.

En relación al segundo ámbito -centrado en su puesta en práctica, seguimiento y consolidación- la principal dificultad en la práctica es que el grupo de docentes responsables del curso (tutores y profesores) tengan una visión compartida tanto del significado y alcance de los RA como de las tareas/actividades, y consecuentes evidencias, que deben/pueden presentar los alumnos para justificar sus avances y progreso. La falta 
de tradición y cultura para trabajar con este enfoque -que busca superar el conocimiento academicista para centrarse en la introducción a la profesión y su progresivo desarrollo-, hace que todavía sean evidentes diferentes interpretaciones de los aportes de los RA, así como de las rúbricas y su aplicación.

Por otra parte, como puede deducirse del proceso explicado, se hace necesario disponer de tiempos y espacios para la coordinación, el diálogo y la negociación con el fin de consensuar los niveles de logro de los RA desde la mirada de las actividades/tareas realizadas en los distintos módulos. Evidentemente, el proceso se complica cuando para cada curso hay más de un grupo con su correspondiente equipo docente pues ello exige coherencia tanto al interior de cada uno de ellos (intra) como entre los distintos equipos docentes (inter).

Lo anterior requiere a su vez liderazgo y coordinación desde el equipo que dirige el proceso, a la par que fomenta una cultura de cambio educativo y ofrece suficientes espacios formativos para que el conjunto de docentes avance de manera coordinada en el marco propuesto, superando la cultura de la educación como transmisión y de la evaluación como calificación presente en parte de los docentes.

Desde la perspectiva de los estudiantes, la primera gran dificultad -paralela a la que evidencian los docentes noveles- es la de "deconstruir" el modelo educativo del que -en su gran mayoría- provienen. Por tanto, una de las primeras tareas es avanzar en el significado y función de conceptos como: competencias, RA, propuestas de trabajo, criterios de evaluación, evidencias... que a la vez que les serán útiles en su desarrollo personal y profesional, son elementos clave en los procesos de evaluación a desarrollar a lo largo del curso.

Por otra parte, cabe destacar la importancia de contar con apoyos tecnológicos que faciliten el trabajo y la reflexión de alumnos y docentes. Como puede ser la herramienta SET (Skills Evolution Tool) creada por Tknika (2015) con la intención de medir la evolución del grado de adquisición de las competencias del alumnado.

En síntesis, subrayamos la importancia de dar tiempo a estos procesos, de consolidar los equipos docentes -a la par que se ofrece formación continua a los mismos en relación con las temáticas que nos ocupan-, y todo ello requiere de un liderazgo claro, comprometido e inclusivo en el que se toma en cuenta y se valora la opinión y participación de todos los implicados en el proceso educativo.

\section{Conclusiones}

Las siguientes conclusiones sintetizan y ponen en relieve la importancia de los RA como elemento central para llevar a cabo una evaluación auténtica del desarrollo de competencias en titulaciones de grado, a la vez que proporcionan algunas claves para transferir la experiencia realizada a otros títulos de grado o máster.

Un cambio profundo de la evaluación de competencias, de modo que esta se realice desde los RA y no desde los instrumentos de evaluación, exige de una sensibilidad, compromiso y acción colectiva de todos los agentes implicados y, especialmente, del equipo directivo y del profesorado. Este componente actitudinal requiere, a la vez, de una conceptualización previa profunda (componente conceptual) que permita comprender, definir y asumir personal y colectivamente un enfoque propio -conjugando la evaluación del y para el 
aprendizaje y la evaluación como aprendizaje y evolución- que derive en un modelo y sistema de evaluación consistente y viable en una formación de calidad.

Un último componente es el procesual, desde el cual debe organizarse y desplegar la implementación del cambio evaluativo. La complejidad propia de este tercer componente debe contemplar acciones eficientes en la aplicación exitosa del modelo y sistema definido. En este sentido la evaluación debe resituarse en el diseño y desarrollo curricular del título, de modo que los RA no sólo sean una concreción finalista de los logros en la adquisición de las competencias, sino que se adopten como referente en el diseño de las actividades de aprendizaje y estén presentes durante su realización dentro y/o fuera del aula. Para ello es imprescindible:

a) Considerar su escalabilidad, que va desde los RA del grado a los niveles de logro en una actividad o propuesta de trabajo específica pasando por la concreción de los RA para cada curso y módulo o asignatura; y

b) Contemplar una evaluación formativa permanente desplegada por los docentes y que, apoyada en la figura del tutor, proporcione retornos cualitativos mediante un feedback basado en las interacciones directas con los estudiantes de modo que se faciliten así mayores niveles de logro en los RA.

Esta redefinición del sistema de evaluación llevado a cabo en los Grados de Educación Infantil y Educación Primaria de HUHEZI no sólo es acorde con el contenido del protocolo de evaluación para la verificación de títulos oficiales en cuanto define y despliega “... un procedimiento general para la valoración de los resultados del aprendizaje de los estudiantes" (REACU, 2011, p. 9), sino que además es una propuesta diferenciada en el contexto universitario español de evaluación de la adquisición de competencias profesionales, en cuanto toda ella está articulada alrededor de los RA.

\section{Referencias}

ANECA [Agencia Nacional de Evaluación de la Calidad y Acreditación] (2013). Guía de apoyo para la redacción, puesta en práctica y evaluación de los resultados del aprendizaje. Madrid: ANECA.

Astigarraga, E. y Carrera, X. (2018). Necesidades a futuro y situación actual de las competencias en Educación Superior en el contex to de España. Revista Digital de Investigación en Docencia Universitaria, 12(2), 35-58. http://doi.org/10.19083/ridu.2018.731

Biggs, J. (2014). Constructive alignment in university teaching. HERDSA Review of Higher Education, 1, 5-22.

Biggs, J. B. y Tang, C. (2011). Teaching for quality learning at university. Maidenhead: McGraw-Hill Education \& Open University Press.

Brown, S. (2015). La evaluación auténtica: el uso de la evaluación para ayudar a los estudiantes a aprender. RELIEVE, 21(2), art M4. Recuperado de https://ojs.uv.es/index.php/RELIEVE/article/view/7674

CEDEFOP [Centro Europeo de Formación Profesional]. (2017). Defining, writing and applying learning outcomes. A European handbook. Recuperado de https://www.cedefop.europa.eu/files/4156_en.pdf

CEDEFOP [Centro Europeo de Formación Profesional]. (2019). Los marcos de cualificaciones en Europa - Evolución en 2018, Promover la confianza mutua a partir de los resultados del aprendizaje. Recuperado de https://www.cedefop.europa.eu/files/9139_es.pdf 
Comisión Europea (2009). El Marco Europeo de Cualificaciones para el aprendizaje permanente (EQF$M E C)$. Luxemburgo: Oficina de Publicaciones de la Unión Europea.

Frey, B. B., Schmitt, V. L. y Allen, J. P. (2012). Defining authentic classroom assessment. Practical Assessment, Research \& Evaluation, 17(2), 1-18.

Real Decreto 1027/2011, de 15 de julio, por el que se establece el Marco Español de Cualificaciones para la Educación Superior. BOE, texto consolidado, última modificación 7 de febrero de 2015. Recuperado de https://www.boe.es/buscar/pdf/2011/BOE-A-2011-13317-consolidado.pdf

Real Decreto 96/2014, de 14 de febrero, por el que se modifican los Reales Decretos 1027/2011, de 15 de julio, por el que se establece el Marco Español de Cualificaciones para la Educación Superior (MECES), y 1393/2007, de 29 de octubre, por el que se establece la ordenación de las enseñanzas universitarias oficiales. Recuperado de https://www.boe.es/boe/dias/2014/03/05/pdfs/BOE-A-2014-2359.pdf

Guerriero, S. (2017). Pedagogical knowledge and the changing nature of the teaching. París: OCDE.

Hattie, J. (2017). "Aprendizaje visible" para profesores. Maximizando el impacto en el aprendizaje. Madrid: Paraninfo.

Hayward, L. (2015). Assessment is learning: the preposition vanishes. Assessment in Education: Principles, Policy \& Practice, 22(1), 27-43. https://doi.org/10.1080/0969594X.2014.984656

Hill, P. y Barber, M. (2014). Preparing for a renaissance in assessment. Nueva York, NY: Pearsons.

Lee, A. M. (2013). Assessment OF ... AS... FOR ... learning. Recuperado de https://annemichellemlee88.wordpress.com/2013/02/14/•assessment-of-as-forlearning/

León, C. (2014). Introducción a la educación superior basada en competencias. Ciudad de México: Limusa.

Ozaeta, A., Mongelos, A., Astigarraga, E. y Garro, E. (2018). Innovando en la universidad. Algunas claves en un proceso de cambio curricular y metodológico. En A. Villa (Ed.), Tendencias actuales de las transformaciones de las universidades en una nueva sociedad digital (pp. 155-166). Bilbao: Foro Internacional de Innovación Universitaria.

Paniagua, A. e Istance, D. (2018). teachers as designers of learning environments: The importance of innovative pedagogies. París: OCDE.

Pellegrino, J. W. (2017). Teaching, learning and assessing 21 st century skills. En S. Guerriero (Ed.), Pedagogical Knowledge and the changing nature of the teaching (pp. 223-251). París: OCDE.

REACU (2011). Evaluación para la Verificación. Protocolo de evaluación para la verificación de títulos universitarios oficiales (Grado y Máster). Recuperado de http://www.aneca.es/Programas-de-evaluacion/Evaluacion-detitulos/VERIFICA/Verificacion-de-Grado-y-Master/Documentacion-y-herramientas

Rundle, N. y Gurney, B. (2017). Constructive alignment diagram. Recuperado de https://elibrary.utas.edu.au/lor/items/27385efo-d 14e-44e7-a5ca-fa6f8e501281/1/

Sarobe, A., López-Salas, N. y Astigarraga, E. (2019). Evaluación del modelo de innovación ETHAZI en los centros de formación profesional de la Comunidad Autónoma del País Vasco. Documento Interno. Eskoriatza: HUHEZI.

SantaCruz, S. (2019). Constructively aligned assessment for deeper learning in Higher Education. Documento Interno - Memoria de Investigación del Personal Investigador en Formación (PIF). Eskoriatza: HUHEZI. 
Scott, C. L. (2015a). El futuro del aprendizaje 2 ¿Qué tipo de aprendizaje se necesita en el siglo XXI? Documentos de Trabajo ERF, $n^{\circ}$ 14. París: UNESCO.

Scott, C. L. (2015b). El futuro del aprendizaje 3 ¿Qué tipo de pedagogías se necesitan en el siglo XXI? Documentos de Trabajo ERF, $\mathrm{n}^{\circ}$. 15. París: UNESCO.

Siarova, H., Sternadel, D. y Mašidlauskaitė, R. (2017). Assessment practices for 21 st century learning: review of evidence. Luxemburgo: Oficina de Publicaciones de la Unión Europea.

Tknika. (2015). SET (Skills Evolution Tool) Evaluación por competencias orientada hacia la evolución. Recuperado de https://tknika.eus/cont/proyectos/set-skills-evolution-tool/\#

Tknika. (2019). Evaluación, feedback y calificación en el trabajo por retos ETHAZI. Recuperado de https://drive.google.com/file/d/1TsN8ls-q6ZESN8wXJ5p92m7nDz-4TZb_/view

\section{Breve Cv de los autores}

\section{Eugenio Astigarraga Echeverría}

Profesor de Educación General Básica. Licenciado en Filosofía y Ciencias de la Educación. Director Pedagógico en la empresa ALECOP, S. Coop. e integrante de la Unidad de Proyectos Educativos Internacionales. Profesor de Grado y Master de la Facultad de Humanidades y Ciencias de la Educación - Mondragon Unibertsitatea. Colaborador de Tknika - Centro de Investigación e Innovación en Formación Profesional. Doctor por Mondragon Unibertsitatea en el Programa en Innovación e Intervención Educativa (Mención Doctorado Internacional). ORCID ID: https://orcid.org/0000-0002-11536420. Email: eastigarraga@mondragon.edu

\section{Arantza Mongelos García}

Doctora en Psicodidáctica por la universidad del País Vasco (UPV-EHU). MSc in Applied Linguistics por la Edinburgh University. Licenciatura en Filología Inglesa (EHU-UPV) Profesora de Grado de la Facultad de Humanidades y Ciencias de la Educación Mondragon Unibertsitatea Coordinadora de Grado de la Facultad de Humanidades y Ciencias de la Educación - Mondragon Unibertsitatea Coordinadora universitaria de proyectos internacionales en MEi (Mondragon Educación Internacional). ORCID ID: https://orcid.org/0000-0001-5795-9173. Email: amongelos@mondragon.edu

\section{Xavier Carrera Farran}

Doctor en Psicopedagogía. Premio extraordinario de Doctorado. Coordinador en la Universidad de Lleida del Programa de Doctorado Interuniversitario en Tecnología Educativa. Coordinador del grupo de investigación COMPETECS (Competencias, Tecnología, Educación y Sociedad) de la Universidad de Lleida [2017 SGR 1700]. Sus líneas de investigación se centran en la Tecnología Educativa; la aplicación de metodologías activas en educación y en la formación basada en competencias. ORCID ID: https://orcid.org/0000-0003-3420-4215. Email: carrera@pip.udl.cat 